\title{
Chaos and Bifurcations of a Leslie-Gower Food Chain with Strong Allee Effect
}

\author{
Chunshan Xue ${ }^{1}$ and Xia Liu ${ }^{2}$ \\ ${ }^{1}$ College of Mathematics and Statistics, Zhoukou Normal University, Zhoukou 466001, China \\ ${ }^{2}$ College of Mathematics and Information Science, Henan Normal University, Xinxiang 453007, China
}

Correspondence should be addressed to Chunshan Xue; 754358042@qq.com

Received 5 August 2015; Revised 29 September 2015; Accepted 1 October 2015

Academic Editor: Luca Guerrini

Copyright (C) 2015 C. Xue and X. Liu. This is an open access article distributed under the Creative Commons Attribution License, which permits unrestricted use, distribution, and reproduction in any medium, provided the original work is properly cited.

\begin{abstract}
Allee effect, as an important biological phenomenon, has been considered in many ecosystems, whereas, its influence on interactions of three or more species is little investigated. In this paper we modify a three-species Leslie-Gower type food chain system by incorporating the strong Allee effect into the source. Our results show that the existence of Allee effect contributes to the occurrence of more complex dynamics of the system, including Hopf, saddle-node, transcritical, saddle-node-Hopf, period-doubling, and period-halving bifurcations and chaos.
\end{abstract}

\section{Introduction}

Stable coexistence of species populations has always been investigated as a focus issue in ecosystems. Recently, several studies reported that in the low density populations the stable coexistence could be influenced easily by Allee effect [1]. Under the mechanism, species have underlying danger to undergo a catastrophic collapse [2,3]. Allee effect, a biological phenomenon of density dependence, has been gradually becoming acknowledged and recognized as a ubiquitous and important mechanism in many theoretical researches of population and community ecology. Therefore, there has been a growing interest in investigating Allee effect [2, 47]. Main attention of some theoretical works related to Allee dynamics is focused on the question that whether interventions of Allee effect to a system have a remarkable influence on dynamics and corresponding evolutions [8-11].

To the best of our knowledge, most of the known literatures devoted to the Allee dynamics are based on the single species or two-component ecosystem $[2,12]$ due to the complexity of high dimension models. However, interactions among community ecology usually involve multispecies. Thus, dynamics investigation of multispecies suffering Allee effects is a meaningful supplement from the point of view of both ecology and mathematics, also for the purpose of exploring the stable coexistence strategies of ecosystem. Research related to Allee effect in three species populations firstly is investigated by [13]. Their efforts were focused on the qualitative aspect. In other words, they primarily address the relativity of the emergent Allee effect and the sizespecific interactions within the prey population. However, the influences of Allee effect on the property of bifurcations and chaos in the multiple population systems have never been considered in detail. Therefore, in the present paper, we will devote our efforts to quantitative aspects of three-species food chain with Allee effects.

Specifically, Allee effect is characterized by the positive correlation between the population density and the total individual fitness usually quantified by the per capita population growth rate and population size or density $[1,7]$. Biological mechanisms giving rise to an Allee effect generally involve social or cooperative processes between multiple individuals [14], for example, mate search difficulty, social facilitation of reproduction, and increased predation risk due to schooling behavior $[1,2,7,14,15]$. There is also another view on the cause of Allee effect. In [14, 16], investigators considered Allee effect as emergent consequences of stage-specific predation. They pointed out that Allee effect came from the shift which a predator induces in the population stage distribution of its prey [14]. Generally, when a single species is subject to 
the Allee effects, its dynamics is governed by the following system:

$$
\dot{X}(t)=X(t) \varphi(X(t)),
$$

where $X(t)$ is population density at time $t$, satisfying $0 \leq$ $X(t) \leq M . \varphi(X)$ presents the per capita growth rate of $X$, and there is a range of $X$ in the interval $[0, M]$, such that

$$
\varphi(0)<\varphi(X)
$$

holds. Generally, (1) has the trivial equilibrium 0 and maximum biological equilibria $X_{2}$ and also admits, at most, one other biological equilibrium $X_{1}$ between 0 and $X_{2}$. Following the definition given by [17], system (1) is identified to have weak Allee effects provided that $(2)$ and $\varphi(0)>0$ hold. Furthermore, a strong Allee effect can occur in system (1) if (2) and $\varphi(0)<0$ hold.

Some theoretical models obeying the definition of (1) are presented and studied solely $[17,18]$ or integrated into the population community $[8,19-21]$. Through above works one can see that the difference between the weak Allee effect and the strong Allee effect is the population growth rate, and the former rises with increasing density; however, the latter does not, which commonly exhibits the alternative stable states or the catastrophic shifts in structure and dynamics corresponding to the bistable or the monostable stages, respectively. Particularly, when system is at the bistable stage, the strong Allee effect introduces a critical population density (or size) above which the particular trophic level of the population will always be present, whereas below which the population will decrease to a low trophic level and even go extinct because the growth rate of the species might become negative $[1,3,14,22]$. When the species suffers a strong Allee effect, some researches indicated that it is apt to have less ability to overcome these additional negative competition effects [23] and thus to be prone to extinction than other species [2, 24]. Obviously, failing to take the strong Allee effect into account could have detrimental consequences for the management and conversation of some natural and exploited populations. This is the exact reason why most works related to Allee dynamics emphasized the investigation of the strong Allee effect $[6-8,20,25]$. These motivate us to study the dynamics induced by strong Allee effect in a multiple species system.

The remaining part of this paper is organized as follows. In the next section, we derive a food chain model with demographic Allee effect [7] caused by considering mate search difficulty of the prey. In Section 3, the existence and the local stability of equilibria of system (5) are discussed. In Section 4, we investigate the bifurcation patterns that system (7) can undergo and chaos behaviors by using the RouthHurwitz rule and some numerical simulations. Finally, a simple discussion is given to conclude this paper.

\section{Model}

In recent times, several two-component prey predator systems in a homogeneous environment with the Allee effects and Leslie-Gower term have been investigated [2, 8, 20, 26], which take the form as

$$
\begin{aligned}
& \dot{X}=X \varphi(X)-Y P(X, Y), \\
& \dot{Y}=Y\left(s-n \frac{Y}{X}\right),
\end{aligned}
$$

where $X \varphi(X)$ obeys the definition of (1) and is a logistic growth rule with an Allee-type function. $P(X, Y)$ is the predator functional response to prey. $n Y / X$ is called the LeslieGower scheme; it is assumed that the growth of the predator with the per capita maximum fertility rate $s$ also follows the logistic type but its environmental carrying capacity depends on the available prey quantity. Such mechanism is also adopted widely in ecosystem modelling [27-32].

In the low density populations, the Allee-type function in (3) can be considered the demographic Allee effect; the cause of that is owing to a reduced ability of females to find mates, which leads to the species growth at a lower rate; for instance, the growth of Glanville fritillary butterfly is reduced due to the difficulty of mating limitation [33]. Mathematically, the biological phenomenon can be displayed by

$$
\dot{x}=x\left(\frac{b_{1} x}{A+x}-d_{1}-c_{0} x\right),
$$

where $A$ is used to quantify the strength of Allee effect; $b_{1}$ and $d_{1}$ are the per capita maximum fertility and death rates of the source. They satisfy $b_{1}>d_{1}$; $c_{0}$ represents the intracompetition intensity of the source population [19]. The growth of the species is assumed to obey the logistic rule but imposed a Michaelis-Menten type function $b_{1} x /(A+x)$. Biologically, the Allee effect $A$ presents a density threshold of population at which the species reaches half of its maximum fertility. By the definitions in (1) and (2), it is easy to infer that system (4) has the strong Allee effect, but not the weak Allee effect. Such Allee effects firstly were presented and used in predator-prey system by [19], who investigate the influence of Allee effect on the ecological and evolutionary conditions that allow for continuously stable strategy and evolutionary branching.

To study Allee dynamics in multiple species, we develop (3), a Leslie-Gower food chain system, by introducing a top predator which consumes the intermediate predator $Y$ to maintain growth itself. The detailed form of the developed systems is described by the following differential equations:

$$
\begin{aligned}
& \dot{x}=x\left(\frac{b_{1} x}{A+x}-d_{1}-c_{0} x\right)-\beta_{0} x y, \\
& \dot{y}=y\left(h_{1}-\frac{c_{1} y}{x}-\frac{\beta_{1} z}{a+y}\right), \\
& \dot{z}=z\left(\frac{\beta_{2} y}{a+y}-d_{2}\right),
\end{aligned}
$$

where $x, y, z$ denote the source, the consumer, and the predator, respectively. Parameters $A, b_{1}, d_{1}, c_{0}, \beta_{0}, h_{1}, c_{1}, \beta_{1}$, $\beta_{2}, d_{2}$, and $a$ are positive, where $c_{1}$ is a quantification of the food supplied by the source; $\beta_{1}$ and $\beta_{2}$ are the capture rate and conversion efficiency. 
A similar system as (5) but without suffering Allee effect has been investigated by [34]. They showed a path along with which system translates its dynamics from Hopf bifurcation to chaos. Compared with the models of [19, 34], system (5) introduces more biological mechanisms; thus, it is more natural to model the interactions of multiple populations, especially for the case of the source at low density. One also can see that system (5) may have more biological steady states due to the existence of the Allee effect. Thus, it is likely to exhibit more complex dynamics which is unclear and needs to be investigated further. On another side, it is different from the works of [19, 34], and we will devote ourselves to address the dynamics analysis of system (5) caused by the Allee mechanism. Our results show that the Allee effect introduced by the low fitness of the source individual facilitates the occurrences of complex bifurcation and chaotic behaviors.

\section{Existence and Stability of Equilibria}

In this section, we focus on the local dynamical behaviors of system (5). In view of biological meaning, we consider system (5) only in $\mathbb{R}_{+}^{3}$ or $\overline{\mathbb{R}_{+}^{3}}$. Clearly, with $(x(0), y(0), z(0))^{T} \in \mathbb{R}_{+}^{3}$, the solutions $(x, y, z)^{T}$ of system (5) are positive for all $t \geqslant 0$.

For simplicity, we begin by taking the scaling

$$
\begin{aligned}
\bar{x} & =\frac{1}{x}, \\
\bar{y}(t) & =\beta_{0} y, \\
\bar{z} & =z, \\
\bar{t} & =b_{1} t
\end{aligned}
$$

to (5), and getting rid of all the bars then the following nondimensional system is obtained:

$$
\begin{aligned}
& \dot{x}=c-\frac{x}{A x+1}+b x+x y, \\
& \dot{y}=h y-\omega x y^{2}-\frac{\beta y z}{m+y}, \\
& \dot{z}=\frac{\alpha y z}{m+y}-d z,
\end{aligned}
$$

where

$$
\begin{aligned}
& c=\frac{c_{0}}{b_{1}}, \\
& b=\frac{d_{1}}{b_{1}} \\
& h=\frac{b_{1} h_{1}}{\beta_{0}^{2}}, \\
& \omega=\frac{c_{1} b_{1}^{2}}{\beta_{0}^{3}},
\end{aligned}
$$

$$
\begin{aligned}
& \beta=\frac{\beta_{1}}{b_{1} \beta_{0}}, \\
& \alpha=\frac{\beta_{2}}{b_{1}}, \\
& d=\frac{d_{2}}{b_{1}}, \\
& m=\frac{a \beta_{0}}{b_{1}} .
\end{aligned}
$$

Then the biological equilibria of system (7) are obtained as follows.

Lemma 1. Assume $0<b<1$. In $\overline{\mathbb{R}_{+}^{3}}$, system (7) may have four boundary equilibria denoted by $E_{0}\left(x_{0}, 0,0\right), \bar{E}_{0}\left(\bar{x}_{0}, 0,0\right)$, $E_{1}\left(x_{1}, y_{1}, 0\right)$, and $\bar{E}_{1}\left(\bar{x}_{1}, \bar{y}_{1}, 0\right)$ if $0<A<A_{0}$ and also may admit two positive $E_{2}\left(x_{2}, y_{2}, z_{2}\right)$ and $\bar{E}_{2}\left(\bar{x}_{2}, \bar{y}_{2}, \bar{z}_{2}\right)$ if $A_{2}<$ $A<A_{1}, \alpha>d, h H-d m \omega \bar{x}_{2}>0$, and $h H-d m \omega x_{2}>0$, where

$$
\begin{aligned}
& A_{0}=\frac{(1-\sqrt{b})^{2} \omega}{c \omega+h} \text {, } \\
& A_{1}=\frac{1}{c}\left(1-\sqrt{b+\frac{m d}{H}}\right)^{2} \text {, } \\
& H=\alpha-d, \\
& \Delta_{1}=(\omega(1-b)-A(h+c \omega))^{2}-4 b A \omega(c \omega+h), \\
& \Delta_{2}=\left(\frac{H(1-c A)}{b H+d m}-1\right)^{2}-\frac{4 A c H}{b H+d m}, \\
& x_{0}=\frac{1-b-c A+\sqrt{(1-b-c A)^{2}-4 c A b}}{2 b A}, \\
& \bar{x}_{0}=\frac{1-b-c A-\sqrt{(1-b-c A)^{2}-4 c A b}}{2 b A}, \\
& x_{1}=\frac{\omega(1-b)-A(h+c \omega)+\sqrt{\Delta_{1}}}{2 b A \omega}, \\
& \bar{x}_{1}=\frac{\omega(1-b)-A(h+c \omega)-\sqrt{\Delta_{1}}}{2 b A \omega}, \\
& y_{1}=\frac{h}{\omega x_{1}}, \\
& \bar{y}_{1}=\frac{h}{\omega \bar{x}_{1}}, \\
& x_{2}=\frac{1}{2}\left(\frac{H(1-c A)}{b H+d m}-1+\sqrt{\Delta_{2}}\right) \text {, } \\
& y_{2}=\frac{d m}{H}
\end{aligned}
$$




$$
\begin{aligned}
& z_{2}=\frac{m \alpha\left(h H-d m \omega x_{2}\right)}{H^{2} \beta}, \\
& \bar{x}_{2}=\frac{1}{2}\left(\frac{H(1-c A)}{b H+d m}-1-\sqrt{\Delta_{2}}\right), \\
& \bar{y}_{2}=\frac{d m}{H} \\
& \bar{z}_{2}=\frac{m \alpha\left(h H-d m \omega \bar{x}_{2}\right)}{H^{2} \beta} .
\end{aligned}
$$

Remark 2. From Lemma 1, we know that system (7) displays multiple equilibria owing to the existence of the strong Allee effect in the source, which is different from the results of [34] (three nonnegative equilibria). In the following analysis, one can see multiple steady states contributing to the occurrence of saddle-node bifurcation. Thus, the system is prone to undergo the catastrophic collapses.

The Jacobian matrix of system (7) at any nonnegative equilibrium $E(X, Y, Z)$ takes the form as follows:

$$
\left(\begin{array}{ccc}
Y+b-\frac{1}{(1+A X)^{2}} & X & 0 \\
-\omega Y^{2} & B_{0} & -\frac{\beta Y}{m+Y} \\
0 & \frac{m \alpha Z}{(m+Y)^{2}} & \frac{\alpha Y}{m+Y}-d
\end{array}\right),
$$

where $B_{0}=h-2 \omega X Y-m \beta Z /(m+Y)^{2}$.

By recurring to estimate the eigenvalues of these equilibria, we have the following results.

Lemma 3. Provided $0<b<1,0<A<A_{0}$, then $E_{0}, \bar{E}_{0}$, and $E_{1}$ are saddles. However, $\bar{E}_{1}$ may be a saddle or node or saddle-focus or focus-node for $b(b-1)(b-3) /\left((b-1)^{2}+2\right)>$ $h>\sqrt{b}-b-c \omega$. More precisely, we get the following:

(i) $\bar{E}_{1}$ is saddle or saddle-focus when $\alpha h /\left(m \omega \bar{x}_{1}+h\right)-d<$ $0,0<A<A^{*}$, and is stable node or focus-node when $\alpha h /\left(m \omega \bar{x}_{1}+h\right)-d<0, A_{1}^{*}<A<\min \left\{A_{2}^{*}, A_{1}\right\}$.

(ii) $\bar{E}_{1}$ is saddle or unstable focus-node when $\alpha \mathrm{h} /\left(m \omega \bar{x}_{1}+\right.$ h) $-d>0,0<A<A^{*}$, and is saddle or saddle-focus when $\alpha h /\left(m \omega \bar{x}_{1}+h\right)-d>0, A_{1}^{*}<A<\min \left\{A_{2}^{*}, A_{1}\right\}$.

$A^{*}, A_{1}^{*}$, and $A_{2}^{*}$ and detailed proof of Lemma 3 can be seen in the Appendix.

Lemma 4. Assume $0<A<A_{1}, \alpha>d$, and $h H-d m \omega \bar{x}_{2}>0$; then $E_{2}$ is unstable, and $\bar{E}_{2}$ is a stable node or stable node-focus for $h H / \bar{x}_{2}(\alpha+d) m<\omega<h H / \bar{x}_{2} d m$.

Proof. The Jacobian matrix of system (7) at equilibrium $E_{2}$ is

$$
M_{E_{2}}=\left(\begin{array}{ccc}
B_{1} & x_{2} & 0 \\
-\omega y_{2}^{2} & C_{1} & -\frac{d \beta}{\alpha} \\
0 & F_{1} & 0
\end{array}\right)
$$

with

$$
\begin{aligned}
& B_{1}=y_{2}+b-\frac{1}{\left(A x_{2}+1\right)^{2}}, \\
& C_{1}=-\frac{(\alpha+d) d m \omega x_{2}}{\alpha H}+\frac{d h}{\alpha}, \\
& F_{1}=\frac{H^{2} z_{2}}{m \alpha} .
\end{aligned}
$$

From Lemma 1, we know that if $E_{2}$ exists, then $F_{1}>0$. Computation shows that $B_{1}$ is a monotonously decreasing function of $A$, in which, together with $\lim _{A \rightarrow A_{1}} B_{1}=0$, we have $B_{1}>0$ for all $0<A<A_{1}$. Hence, the determinant of $M_{E_{2}}$ denoted by $\operatorname{det} M_{E_{2}}=-d B_{1} F_{1} / \alpha$ is negative, which implies that $E_{2}$ is a saddle or a saddle-focus or an unstable node.

Similarly, the Jacobian matrix of system (7) at equilibrium $\bar{E}_{2}$ is

$$
M_{\bar{E}_{2}}=\left(\begin{array}{ccc}
B_{2} & \bar{x}_{2} & 0 \\
-\omega \bar{y}_{2}^{2} & C_{2} & -\frac{d \beta}{\alpha} \\
0 & F_{2} & 0
\end{array}\right) \text {, }
$$

with

$$
\begin{aligned}
& B_{2}=\bar{y}_{2}+b-\frac{1}{\left(A \bar{x}_{2}+1\right)^{2}}, \\
& C_{2}=-\frac{(\alpha+d) d m \omega \bar{x}_{2}}{H \alpha}+\frac{d h}{\alpha}, \\
& F_{2}=\frac{H^{2} \bar{z}_{2}}{m \alpha} .
\end{aligned}
$$

The characteristic equation of $M_{E_{2}}$ is

$$
\lambda^{3}+G_{1} \lambda^{2}+G_{2} \lambda+G_{3}=0,
$$

where

$$
\begin{aligned}
& G_{1}=-C_{2}-B_{2}, \\
& G_{2}=\frac{d F_{2} \beta}{\alpha}+\omega \bar{x}_{2} y_{2}^{2}+C_{2} B_{2}, \\
& G_{3}=-\frac{d F_{2} B_{2} \beta}{\alpha} .
\end{aligned}
$$

In order to use the Routh-Hurwitz criterion, we get

$$
\begin{aligned}
G_{1} G_{2}-G_{3} & =-\frac{d C_{2} F_{2} \beta}{\alpha}-\left(C_{2}+B_{2}\right)\left(\omega \bar{y}_{2}^{2} \bar{x}_{2}+C_{2} B_{2}\right) \\
& \triangleq U(\omega) .
\end{aligned}
$$

By computation, we infer that $B_{2}<0$ for $0<A<A_{1}$ and $\mathrm{C}_{2}<0$ for

$$
\frac{h H}{\bar{x}_{2}(\alpha+d) m}<\omega<\frac{h H}{\bar{x}_{2} d m} .
$$

Therefore, $G_{1}>0, G_{2}>0, G_{3}>0$, and $G_{1} G_{2}-G_{3}>0$ if $0<A<A_{1}$ and (18) hold, which indicates that $\bar{E}_{2}$ is stable node or stable node-focus. 


\section{Results}

4.1. Hopf Bifurcation Analysis. From Lemma 4 and theory developed by Liu [35], we derive that system (7) can undergo the Hopf bifurcation at $\bar{E}_{2}$ if there exists $\omega_{*} \in\left(0, h H / \bar{x}_{2} d m\right)$ and satisfies that

$$
\begin{aligned}
& G_{1}\left(\omega_{*}\right)>0, \\
& G_{3}\left(\omega_{*}\right)>0, \\
& G_{1}\left(\omega_{*}\right) G_{2}\left(\omega_{*}\right)-G_{3}\left(\omega_{*}\right)=0, \\
&\left.\frac{\mathrm{d} U(\omega)}{\mathrm{d} \omega}\right|_{\omega=\omega_{*}} \neq 0 .
\end{aligned}
$$

Firstly, we notice that $G_{1}>0$ if

$$
\omega>\frac{\left(h d+B_{2} \alpha\right) H}{\bar{x}_{2}(\alpha+d) d m}
$$

holds. Therefore, if $\omega_{*}$ exists, then $\omega_{*} \in(\max \{0,(h d+$ $\left.\left.\left.B_{2} \alpha\right) H / \bar{x}_{2}(\alpha+d) d m\right\}, h H / \bar{x}_{2}(\alpha+d) m\right] . U(\omega)$ can be rewritten as a quadratic function of $\omega$, and

$$
\begin{gathered}
\left.U(\omega)\right|_{\omega=h H / \bar{x}_{2}(\alpha+d) m}=-\frac{B_{2} h H \bar{y}_{2}^{2}}{m(\alpha+d)}>0, \\
\lim _{\omega \rightarrow\left(h d+B_{2} \alpha\right) H / \bar{x}_{2}(\alpha+d) d m} U(\omega)=\frac{B_{2} d \beta}{\alpha}<0, \\
\lim _{\omega \rightarrow 0} U(\omega)=\frac{h d\left(d \beta+h d B_{2}+B_{2}^{2} \alpha\right)}{-\alpha^{2}} .
\end{gathered}
$$

A simple computation shows that $-h d\left(d \beta+h d B_{2}\right) / \alpha^{2}<0$ for $0<A<A_{1}$; thus, $\lim _{\omega \rightarrow 0} U(\omega)=-h d\left(d \beta+h d B_{2}+\right.$ $\left.B_{2}^{2} \alpha\right) / \alpha^{2}<-h d\left(d \beta+h d B_{2}\right) / \alpha^{2}<0$. That is, (21) implies that $\omega_{*}$ exists and satisfies $G_{1}\left(\omega_{*}\right)>0, G_{3}\left(\omega_{*}\right)>0, C_{2}\left(\omega_{*}\right)>0$, and $U\left(\omega_{*}\right)=0$. Also notice that $G_{1}\left(\omega_{*}\right) G_{2}\left(\omega_{*}\right)=G_{3}\left(\omega_{*}\right)$, such that $G_{2}\left(\omega_{*}\right)>0$.

Secondly, the transverse condition

$$
\begin{aligned}
& \left.\frac{\mathrm{d} U(\omega)}{\mathrm{d} \omega}\right|_{\omega=\omega_{*}} \\
& =\frac{d^{2} m \bar{x}_{2} C_{2}\left(\omega_{*}\right)}{\alpha} \\
& \quad+\frac{(\alpha+d) d m \bar{x}_{2}}{H \alpha}\left[-G_{1}\left(\omega_{*}\right) B_{2}+G_{2}\left(\omega_{*}\right)\right] \\
& \quad+G_{1}\left(\omega_{*}\right) \bar{x}_{2} \bar{y}_{2}^{2}>0 .
\end{aligned}
$$

Without losing the generality, we set $\omega_{*}=\max \{\omega: U(\omega)=$ $\left.0,0<\omega<h H / \bar{x}_{2} d m\right\}$; thus, together with the above analysis and Lemma 4 , we have the following result.

Theorem 5. Set $0<A<A_{1}, H>0$, and $0<\omega<$ $h H / \bar{x}_{2} d m$; then there is a $\omega_{*} \in\left(\max \left\{0,\left(h d+B_{2} \alpha\right) H / \bar{x}_{2}(\alpha+\right.\right.$ d)dm\}, $\left.h H /\left(\bar{x}_{2} \alpha+d\right) m\right)$, so that equilibrium $\bar{E}_{2}$ is local asymptotically stable when $\omega_{*}<\omega<h H / \bar{x}_{2} d m$ and unstable when $0<\omega<\omega_{*}$. Furthermore, a Hopf bifurcation occurs when $\omega=\omega_{*}$.
Remark 6. In Theorem 5, we choose $\omega$ as bifurcation parameter. When $\omega$ approaches $\omega_{*}$, a stable periodic solution is bifurcated from equilibrium $\bar{E}_{2}$. From Figure 1(a), we can see that the value of the source $\bar{x}_{2}$, except for its stability, does not vary with $\omega$ changing continuously. However, if we take the Allee effect $A$ as bifurcation parameter, in Figure 1(b), we discover that the value and stability of $\bar{x}_{2}$ will change with $A$ varying. These indicate that the Allee effect $A$ has the bigger impacts than $\omega$ on the dynamics of system (7).

4.2. Dynamics Evolution. To completely know the dynamics induced by the combined action of the Allee effect and other parameters, in the following, we investigate the dynamical behavior evolution of system (7) by recurring to the numerical methods.

Firstly, the Hopf bifurcation as shown in Figure 1(a) occurs at $\omega \approx 7.78$, which forces three species to shift their states from the stable coexistence to periodical cycle as $\omega$ decreasing continuously. However, the period-1 behavior will be translated to period- 2 cycle with a period-doubling bifurcation appearing at $\omega \approx 3.57$. Subsequently, the perioddoubling cascade happens with $\omega$ decreasing which may lead to the chaos behavior of system (7); for instance, when $\omega=$ 2.0, system (7) exhibits a chaos attractor (see Figure 2(a)).

Similar result of Hopf bifurcation also is achieved by the Allee effect $A$. As shown in Figure 1(b), we see that system (7) undergoes the Hopf bifurcation when $A \approx 0.00508$. In addition, from Lemma 4 , we also see that under the condition $0<A<A_{1}, h H-d m \omega \bar{x}_{2}>0$, and $h H-d m \omega x_{2}>$ 0 two equilibria $\bar{E}_{2}$ and $E_{2}$ coexist, one is stable and the other is unstable. Furthermore, $\bar{E}_{2}$ and $E_{2}$ collide when $A=$ $A_{1}$, which signifies that a saddle-node bifurcation occurs (see Figure 1(b)). Similar arguments can be implemented to equilibria $\bar{E}_{1}$ and $E_{1}$; there also is a saddle-node bifurcation at $A=A_{0}$.

System parameters are chosen as $A=0.01, m=0.3$, $b=0.2, d=0.8, c=0.2, \beta=2.4, \alpha=2.4, h=1.2$, and $\omega=0.6$ such that system (7) has a positive equilibrium as the saddle-focus with a stable one-dimensional manifold $W_{\text {loc }}^{s}$ and an unstable two-dimensional manifold $W_{\text {loc }}^{u}$ and a boundary equilibrium as the saddle; then we can see that there is a stable limit cycle bifurcating from the homoclinic bifurcation as shown in Figure 3.

Finally, from Lemmas 3 and 4, we can exhibit the existence of transcritical bifurcation associated with equilibria $\bar{E}_{1}$ and $\bar{E}_{2}$. Figure $4\left(\right.$ a) shows that $\bar{E}_{2}$ and $\bar{E}_{1}$ get close to each other with $\omega$ increasing and coincide and exchange their stabilities at $\omega \approx 4.16$ and subsequently separate into two different steady states.

Remark 7. From the above numerical examples, one can see that system (7) may display Hopf, saddle-node, homoclinic, and transcritical bifurcations with parameters $A$ and $\omega$ changing. Moreover, system (7) also can undergo other degenerated bifurcations, besides the saddle-node bifurcation, for example, saddle-node-Hopf bifurcation. It is easy to check that the characteristic polynomial corresponding to 


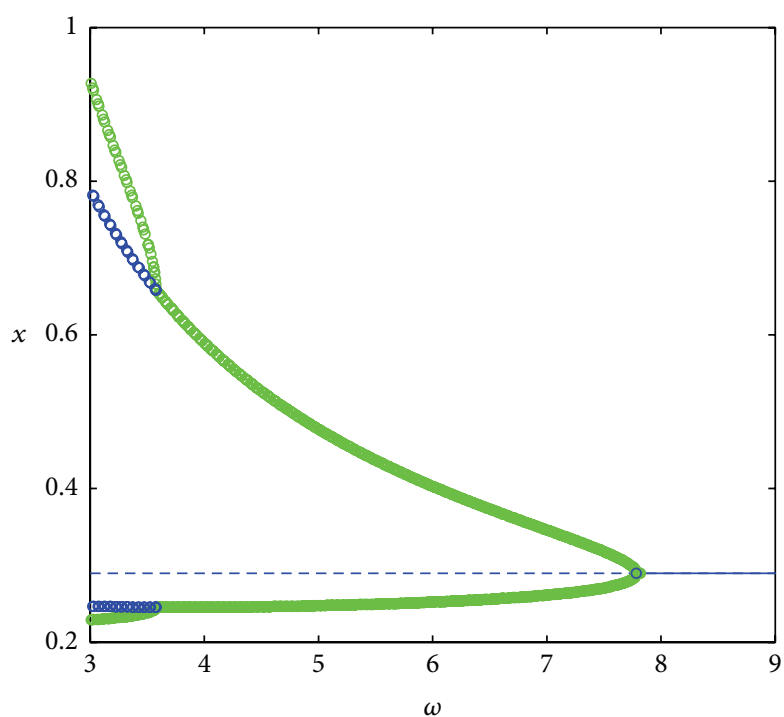

(a)

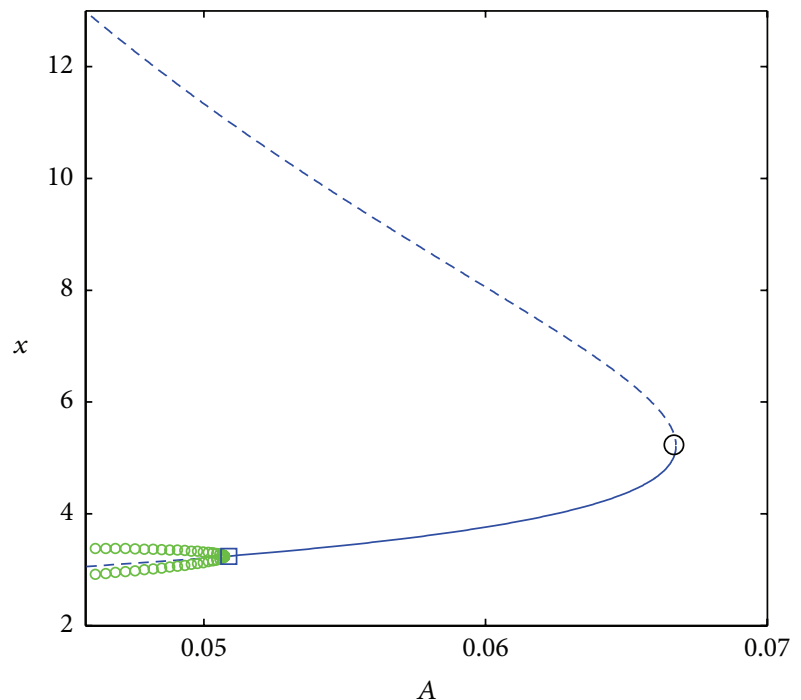

(b)

Figure 1: Hopf bifurcation induced, respectively, by $\omega$ and $A$. (a) Hopf and period-doubling bifurcations are exhibited with $3<\omega<9$, $A=0.2, b=0.5, c=0.1, d=0.2, h=1.0, \beta=2, \alpha=0.6$, and $m=0.2$. (b) Hopf and saddle-node bifurcations when $0.045<A<0.07$, $\omega=1.0, b=0.4, c=1, d=0.6, h=2.0, \beta=2.6, \alpha=1.8$, and $m=0.3$ where the black square denotes saddle-node bifurcation. The blue solid and the dash lines denote stable and unstable states, respectively. The green and blue circle lines are used to mark the periodic solution bifurcating from $\bar{E}_{2}$.

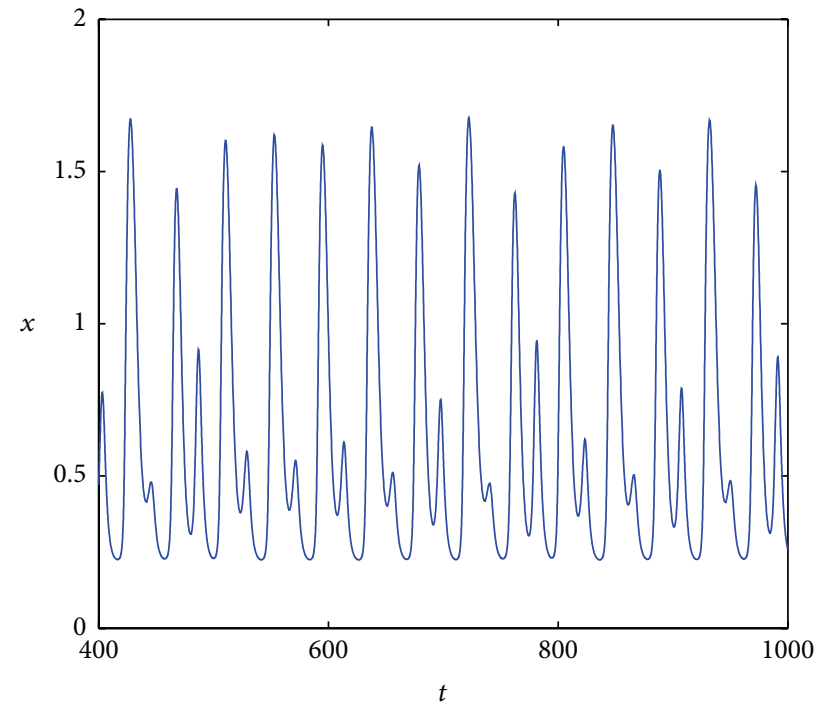

(a)

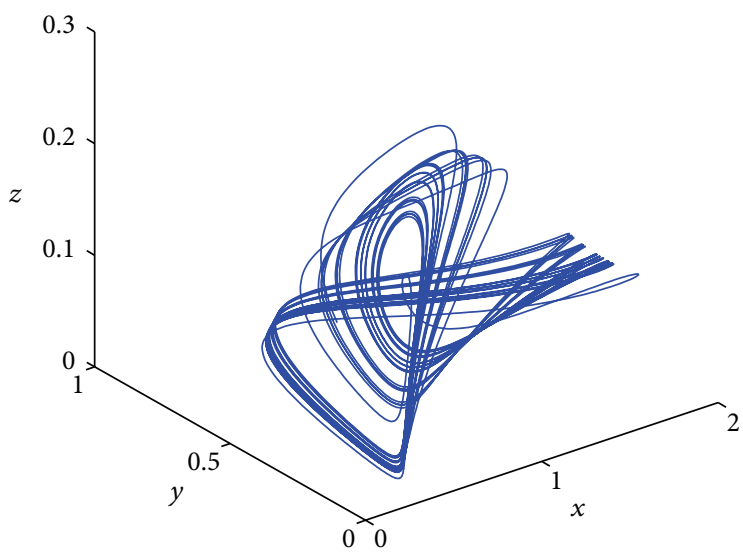

(b)

Figure 2: Schematic diagram of a chaos attractor when $\omega=2.0, A=0.2, b=0.5, c=0.1, d=0.2, h=1.0, \beta=2, \alpha=0.6$, and $m=0.2$. (a) The time evolution of resource $x$. (b) The 3D phase of the attractor.

the Jacobian matrix (13) has a zero eigenvalue when $A=A_{1}$. If we take

$\omega$

$$
=\frac{h(b H+d m)(b H+d m+H-2 \sqrt{H(b H+d m)})}{c m(2 d+H)(\sqrt{H(b H+d m)}-b H-d m)},
$$

then matrix (13) also has a pair of purely imaginary eigenvalues. These imply that system (7) may result in the saddlenode-Hopf bifurcation which is verified in Figure 4(b) (the intersection of the Hopf bifurcation curve and the saddlenode curve).

4.3. Chaotic Behavior. It is known from the published theoretical works that ecological chaos may have prominent 


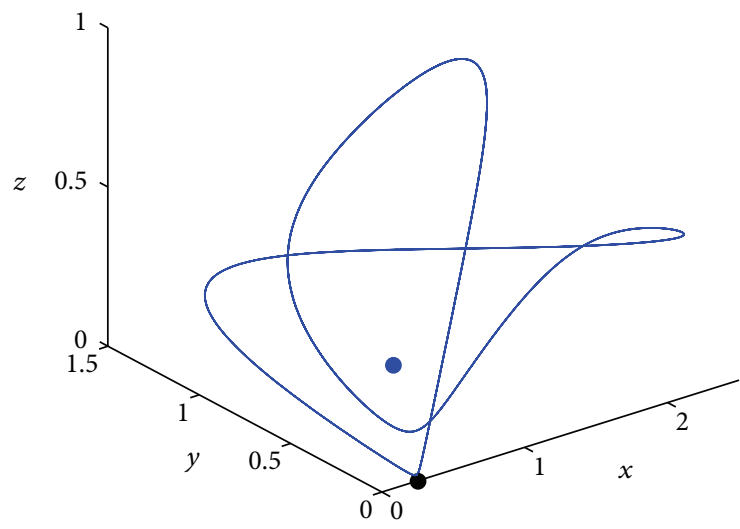

Figure 3: Stable limit cycle is shown when $A=0.01, m=0.4, b=$ $0.2, d=0.8, c=0.2, \beta=2.4, \alpha=2.4, h=1.2$, and $\omega=0.6$, where black solid dot is a saddle and the blue solid dot is a saddle focus.

influences on the population and community dynamics. Thus, how to identify the factors potentially facilitating or inhibiting chaotic behavior is an important but challenging subject. In what follows, extensive numerical simulations are carried out for various values of parameters and for different sets of initial conditions. We choose $A, c$, and $\omega$ as bifurcation parameters, respectively, and keep other parameters fixed.

Firstly, to get a deep insight into the complex dynamics induced by the Allee effect, we implement the numerical simulation in plane $(A, x)$. Figure 5 exhibits a route along with which system (7) transforms its dynamical behaviors from period- $n$ attractors $(n=2,4, \ldots)$ to chaos motion and to period- $n$ attractor through some period-doubling and period-halving sequences, respectively. More precisely, with $A$ increasing, a period-doubling bifurcation occurs at $A \approx$ 0.0305 , which forces system (7) to go to a period- 4 cycle. Subsequent period-doubling cascade brings the system to the chaotic motion with $A$ increasing continuously.

On the contrary, the chaotic motion can inversely switch to period- $n$ state through several period-halving bifurcations. It is known that these chaotic behaviors displayed in system (7) heavily depend on the initial states of three species or the perturbation coming from the external stimulations. That is, the different dynamical regimes are likely to be exhibited at the same parameter value. For instance, choosing $A=$ 0.1651 , system (7) may undergo a period-2 cycle when $(x(0), y(0), z(0))=(3.825,0.356,0.323)$ or a quasiperiodic behavior when $(x(0), y(0), z(0))=(0.825,0.356,0.323)$ (see Figure 6) which is the most intuitive definition of chaos.

To deeply understand the combined effect of the Allee effect and other parameters, similar effects are produced by an increase in parameters $c$ and $\omega$, respectively (see Figures 7 and 9).

\section{Conclusions and Discussion}

The complexities of ecosystems, such as multiple bifurcations and chaotic behaviors, have been a focus of intense research in the ecologic and mathematics fields for the purpose of investigating how to keep the persistent coexistence of species populations in community $[30,36]$. Such studies of population interaction based on mathematical models have provided valuable insight into ecological dynamics. Recently, several works were devoted to address the influence of Allee effect on dynamics of population to explore the underlying mechanism of regulating stable coexistence of species in community.

In the present paper, we consider the Allee effect for the prey (the resource) in a modified Leslie-Gower food chain system. Some main properties including Hopf, perioddoubling, saddle-node, transcritical, and saddle-node-Hopf bifurcations and chaotic behaviors are studied qualitatively and quantitatively. Results based on our analysis and numerical simulations show that the Allee effect not only increases the complexity of the system structure but also promotes the occurrence of chaotic behaviors. As shown in Figures 7 and 8, system displays period-doubling cascade, chaos motion, and period-halving cascade when interfered by the Allee effect (see Figure 7), whereas it only keeps period-2 and period-4 cycles when there is no disturbance of the Allee effect (see Figure 8).

\section{Appendix}

\section{The Proof of Lemma 3}

Proof. Substituting $E_{0}$ and $\bar{E}_{0}$ into (10), respectively, one can show that both $M_{E_{0}}$ and $M_{\bar{E}_{0}}$ have eigenvalues $h$ and $-d$; thus, $E_{0}$ and $\bar{E}_{0}$ are saddles.

Jacobian matrix at $E_{1}$ is

$$
M_{E_{1}}=\left(\begin{array}{ccc}
L_{0} & x_{1} & 0 \\
-\frac{h^{2}}{\omega x_{1}^{2}} & -h & -\frac{\beta h}{m \omega x_{1}+h} \\
0 & 0 & \frac{\alpha h}{m \omega x_{1}+h}-d
\end{array}\right)
$$

where $L_{0}=h / \omega x_{1}+b-1 /\left(1+A x_{1}\right)^{2}$.

Obviously, one eigenvalue of $M_{E_{1}}$ is $\alpha h /\left(m \omega x_{1}+h\right)-d$; others are determined by the matrix

$$
M_{E_{1}(2 \times 2)}=\left(\begin{array}{cc}
\frac{h}{\omega x_{1}}+b-\frac{1}{\left(1+A x_{1}\right)^{2}} & x_{1} \\
-\frac{h^{2}}{\omega x_{1}^{2}} & -h
\end{array}\right) .
$$

Computation shows that the determinant of (A.2) satisfies $-h\left(b-1 /\left(x_{1} A+1\right)^{2}\right)<0$ with the conditions $0<b<1$ and $0<A<A_{0}$, which implies that matrix (A.2) has two real eigenvalues but has opposite signs. That is, $E_{1}$ is a saddle.

Similarly we also carry out investigation for the eigenvalues of $M_{\bar{E}_{1}}$, one is $\alpha h /\left(m \omega \bar{x}_{1}+h\right)-d$, and others may be two real roots with the same signs or be a pair of conjugate complex numbers since the determinant of $M_{\bar{E}_{1}(2 \times 2)}$ satisfies that $-h\left(b-1 /\left(\bar{x}_{1} A+1\right)^{2}\right)>0$. The trace of $M_{\bar{E}_{1}(2 \times 2)}$ denoted by $\operatorname{Tr}_{1}(A) \triangleq h / \omega \bar{x}_{1}-h+b-1 /\left(A \bar{x}_{1}+1\right)^{2}$ can be regarded as 


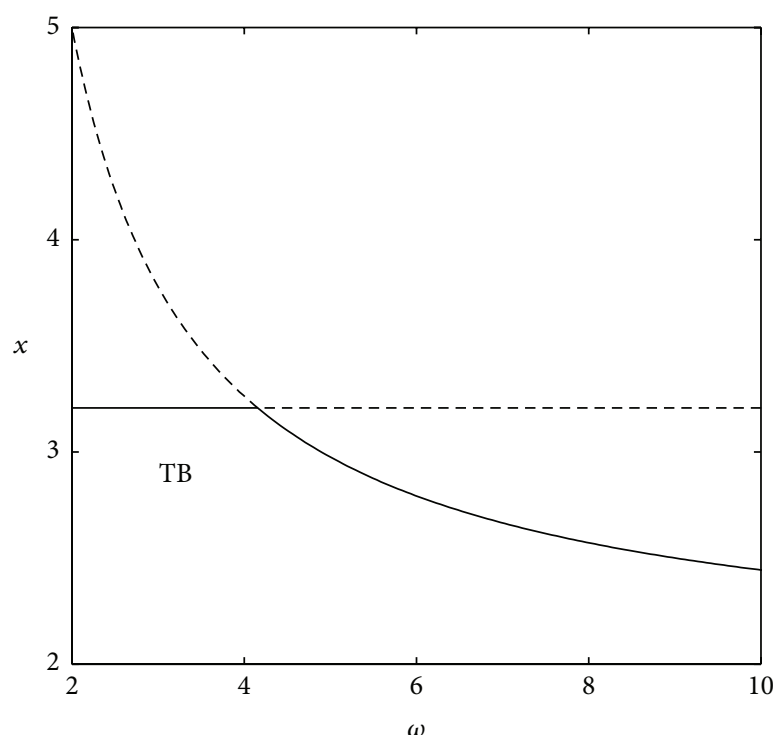

(a)

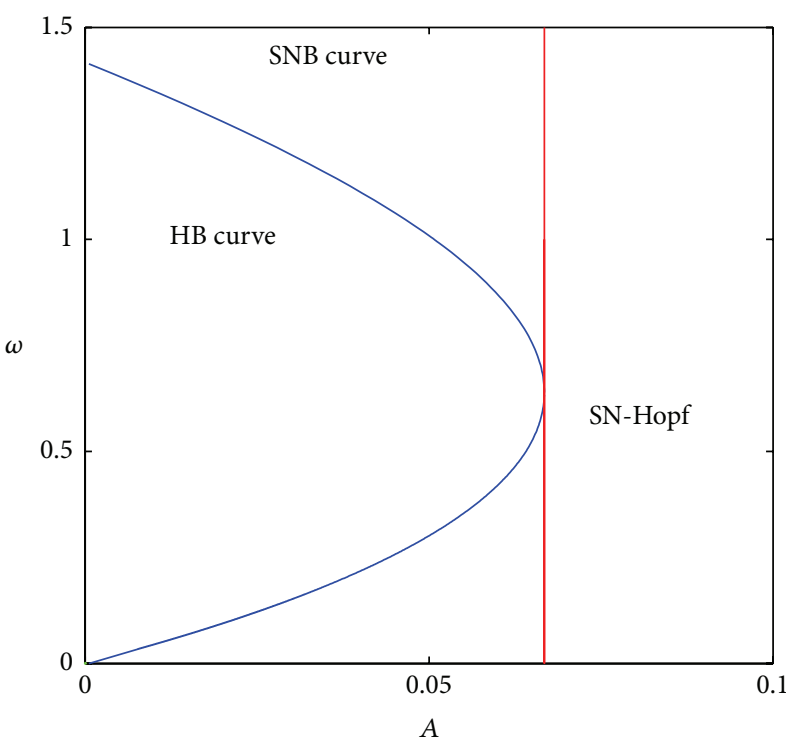

(b)

Figure 4: Transcritical and saddle-node-Hopf bifurcations of system (7). (a) A transcritical bifurcation occurs when equilibria $\bar{E}_{2}$ and $\bar{E}_{1}$ collide at $\omega \approx 4.157$, where $A=0.05, b=0.4, c=1, d=0.6, h=2.0, \beta=2.6, \alpha=1.8$, and $m=0.3$, where the black dash curve denotes stable equilibrium and the black solid curve denotes unstable equilibrium. (b) The double parameter bifurcation in the plane $(A, \omega)$ and other parameters take the same values as in (a), where the blue solid curve is Hopf bifurcation curve (HB) and the red solid line is saddle-node bifurcation curve. The intersection of $\mathrm{HB}$ curve and SNB curve is saddle-node-Hopf bifurcation (SN-Hopf).

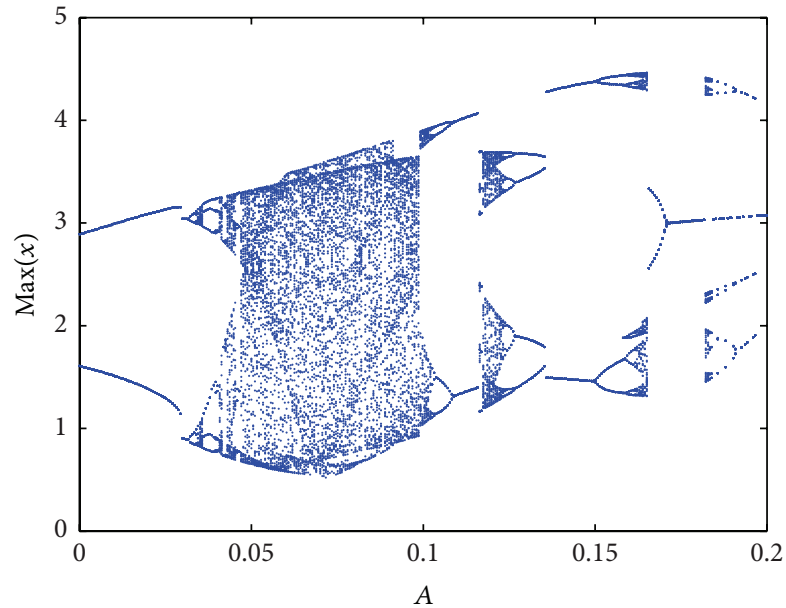

FIGURE 5: The bifurcation diagram in plane $(A, x)$ with $0<A<0.2$, $m=0.3, b=0.2, d=0.8, c=0.25, \beta=2.4, \alpha=1.7, h=1.2$, and $\omega=0.6$.

a continuous function of variable $A$ for $0<A<A_{0}$. Hence, if

$$
\begin{gathered}
\lim _{A \rightarrow 0^{+}} \operatorname{Tr}_{1}(A)<0, \\
\lim _{A \rightarrow A_{0}^{-}} \operatorname{Tr}_{1}(A)<0, \\
\text { or } \lim _{A \rightarrow 0^{+}} \operatorname{Tr}_{1}(A)>0, \\
\lim _{A \rightarrow A_{0}^{-}} \operatorname{Tr}_{1}(A)<0,
\end{gathered}
$$

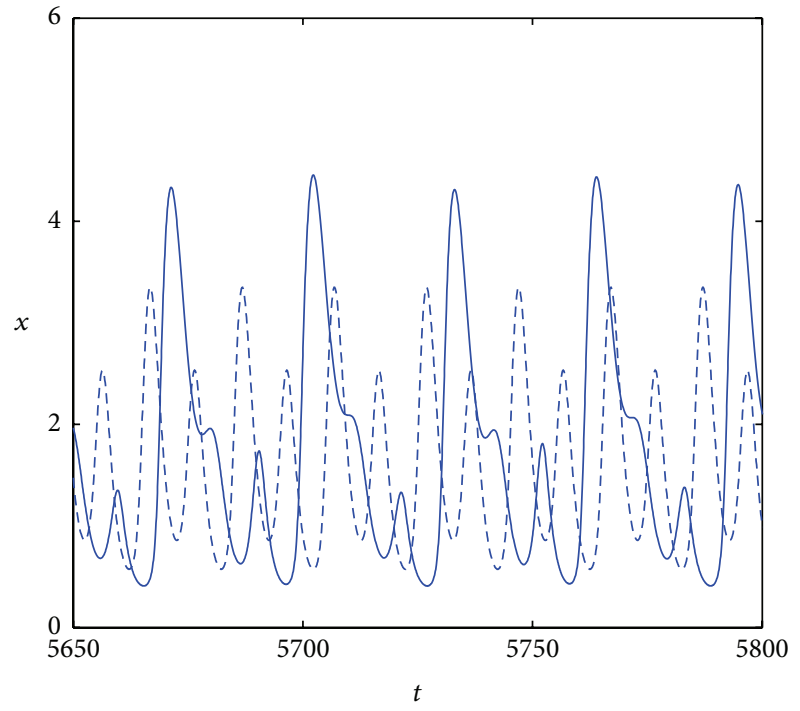

FIGURE 6: The time evolution of resource $x$ when $A=0.1651$, $m=0.3, b=0.2, d=0.8, c=0.25, \beta=2.4, \alpha=1.7, h=1.2$, and $\omega=$ 0.6 , where the cases of blue solid and dash lines are corresponding to initial conditions $(3.825,0.356,0.323)$ and $(0.825,0.356,0.323)$, respectively.

then there exists at least one $A^{*} \in\left(0, A_{0}\right)$ so that $\operatorname{Tr}_{1}\left(A^{*}\right)=0$. Equation (A.3) is equivalent to

$$
(\sqrt{b}-b-c \omega-h)\left(\frac{b(1-b)(3-b)}{(1-b)^{2}+2}-h\right)<0 .
$$




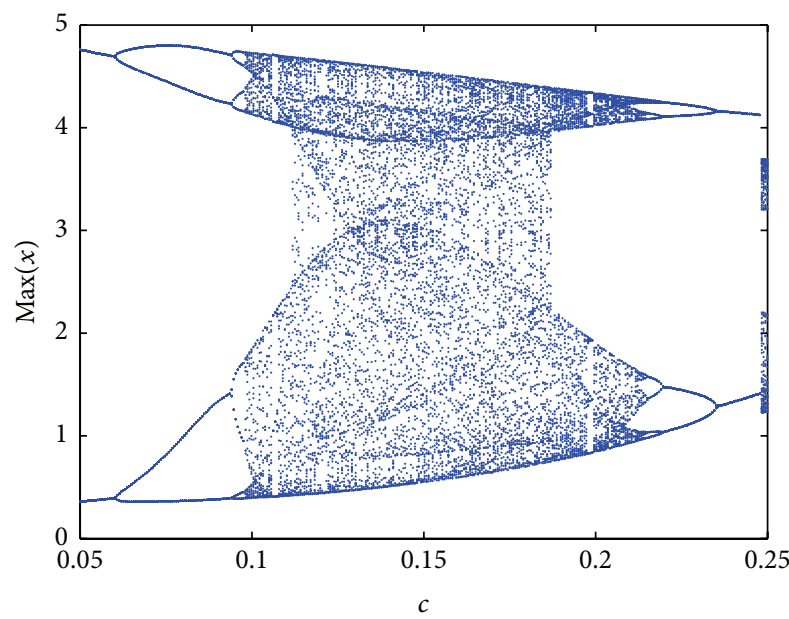

Figure 7: The bifurcation diagram of system (7) in plane $(c, x)$ when $0.05 \leqslant c \leqslant 0.25, A=0.12, m=0.3, b=0.2, d=0.8, \beta=2.4, \alpha=1.7$, $h=1.2$, and $\omega=0.6$.

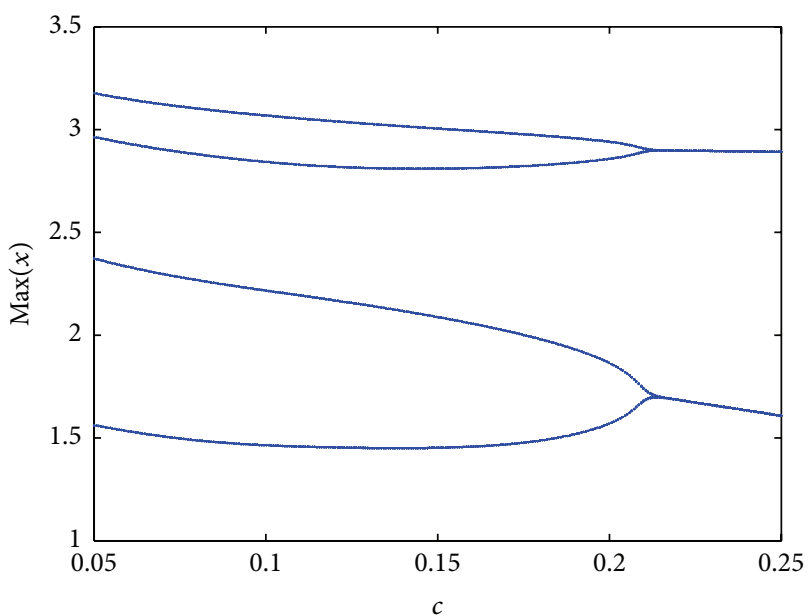

FIGURE 8: The bifurcation diagram in plane $c, x$ with $0.05 \leqslant c \leqslant 0.25$, $A=0, m=0.3, b=0.2, d=0.8, \beta=2.4, \alpha=1.7, h=1.2$, and $\omega=0.6$.

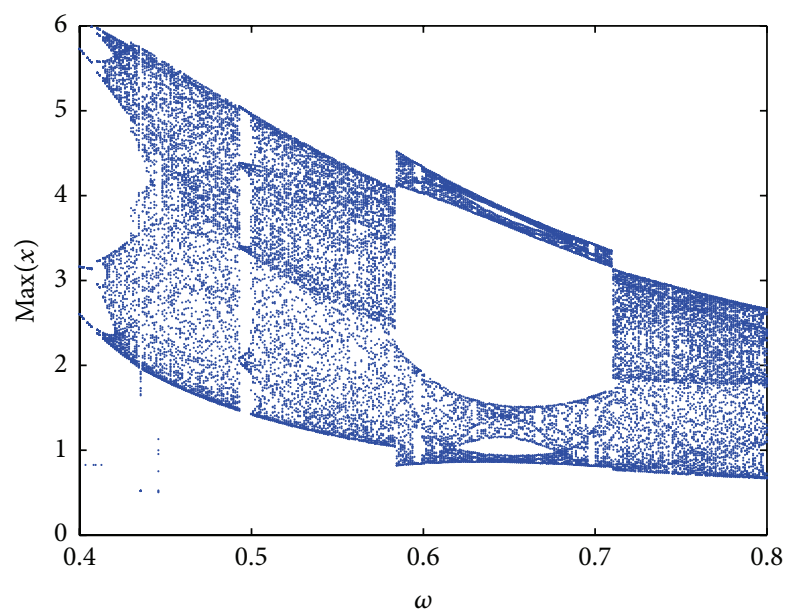

FIgURE 9: The bifurcation diagram of system (7) in plane $(\omega, x)$ when $0.4 \leqslant \omega \leqslant 0.8, A=0.12, m=0.3, c=0.2, b=0.2, d=0.8, \beta=2.4$, $\alpha=1.7, h=1.2$, and $\omega=0.6$.
Notice that $b(1-b)(3-b) /\left((1-b)^{2}+2\right)>\sqrt{b}-b-c \omega$ for $0<b<1$; hence (A.4) can be rewritten as

$$
\sqrt{b}-b-c \omega<h<\frac{b(1-b)(3-b)}{(1-b)^{2}+2} .
$$

Define $S \triangleq\left\{A \mid \operatorname{Tr}_{1}(A)=0\right\}$. Without loss of generality, set $A_{1}^{*}=\min \{S\}$ and $A_{2}^{*}=\min \left\{S \backslash A_{1}^{*}\right\}$. It follows from (A.5) that $\bar{E}_{1}$ is unstable if $0<A<A_{1}^{*}$ and stable if $A_{1}^{*}<A<$ $\min \left\{A_{2}^{*}, A_{1}\right\}$ and $\alpha h /\left(m \omega \bar{x}_{1}+h\right)-d<0$. To sum up the above analysis, we can attain the results of Lemma 3.

\section{Conflict of Interests}

The authors declare that there is no conflict of interests regarding the publication of this paper.

\section{Acknowledgments}

The authors would like to thank the editor and the anonymous reviewers for their constructive suggestions and comments, which improved the presentation of the paper.

\section{References}

[1] W. C. Allee, Animal Aggregations: A Study in General Sociology, University of Chicago Press, Chicago, Ill, USA, 1931.

[2] P. Aguirre, E. Gonzlez-Olivares, and E. E. Sáez, "Three limit cycles in a Leslie-Gower predator-prey model with additive Allee effect," SIAM Journal on Applied Mathematics, vol. 69, no. 5, pp. 1244-1262, 2009.

[3] J. Bascompte, "Extinction thresholds: insights from simple models," Annales Zoologici Fennici, vol. 40, no. 2, pp. 99-114, 2003.

[4] W. Wang, Y.-N. Zhu, Y. C. Cai, and W. J. Wang, "Dynamical complexity induced by Allee effect in a predator-prey model," Nonlinear Analysis: Real World Applications, vol. 16, no. 1, pp. 103-119, 2014.

[5] D. Hadjiavgousti and S. Ichtiaroglou, "Allee effect in a preypredator system," Chaos, Solitons \& Fractals, vol. 36, no. 2, pp. 334-342, 2008.

[6] F. Courchamp, L. Berec, and J. Gascoigne, Allee Effects in Ecology and Conservation, Oxford University Press, Oxford, UK, 2008.

[7] L. Berec, E. Angulo, and F. Courchamp, "Multiple Allee effects and population management," Trends in Ecology and Evolution, vol. 22, no. 4, pp. 185-191, 2007.

[8] P. Aguirre, J. D. Flores, and E. González-Olivares, "Bifurcations and global dynamics in a predator-prey model with a strong Allee effect on the prey, and a ratio-dependent functional response," Nonlinear Analysis. Real World Applications, vol. 16, no. 1, pp. 235-249, 2014.

[9] W.-X. Wang, Y.-B. Zhang, and C.-Z. Liu, "Analysis of a discretetime predator-prey system with Allee effect," Ecological Complexity, vol. 8, no. 1, pp. 81-85, 2011.

[10] H. T. M. Eskola and K. Parvinen, "On the mechanistic underpinning of discrete-time population models with Allee effect," Theoretical Population Biology, vol. 72, no. 1, pp. 41-51, 2007.

[11] S.-R. Zhou, Y.-F. Liu, and G. Wang, "The stability of predatorprey systems subject to the Allee effects," Theoretical Population Biology, vol. 67, no. 1, pp. 23-31, 2005. 
[12] C. Çelik and O. Duman, "Allee effect in a discrete-time predatorprey system," Chaos, Solitons and Fractals, vol. 40, no. 4, pp. 1956-1962, 2009.

[13] A. M. de Roos and L. Persson, "Size-dependent life-history traits promote catastrophic collapses of top predators," Proceedings of the National Academy of Sciences of the United States of America, vol. 99, no. 20, pp. 12907-12912, 2002.

[14] A. M. de Roos, L. Persson, and H. R. Thieme, "Emergent Allee effects in top predators feeding on structured prey populations," Proceedings of the Royal Society B: Biological Sciences, vol. 270, no. 1515, pp. 611-618, 2003.

[15] C. E. Brassil, "Mean time to extinction of a metapopulation with an Allee effect," Ecological Modelling, vol. 143, no. 1-2, pp. 9-16, 2001.

[16] T. van Kooten, A. M. de Roos, and L. Persson, "Bistability and an Allee effect as emergent consequences of stage-specific predation," Journal of Theoretical Biology, vol. 237, no. 1, pp. 6774, 2005.

[17] M.-H. Wang and M. Kot, "Speeds of invasion in a model with strong or weak Allee effects," Mathematical Biosciences, vol. 171, no. 1, pp. 83-97, 2001.

[18] B. Dennis, "Allee effects: population growth, critical density, and the chance of extinction," Natural Resource Modeling, vol. 3, no. 4, pp. 481-538, 1989.

[19] J. Zu, M. Mimura, and J. Y. Wakano, "The evolution of phenotypic traits in a predator-prey system subject to Allee effect," Journal of Theoretical Biology, vol. 262, no. 3, pp. 528543, 2010.

[20] P. Aguirre, E. González-Olivares, and E. E. Sáez, “Two limit cycles in a Leslie-Gower predator-prey model with additive Allee effect," Nonlinear Analysis. Real World Applications, vol. 10, no. 3, pp. 1401-1416, 2009.

[21] M. Sen, M. Banerjee, and A. Morozov, "Bifurcation analysis of a ratio-dependent prey-predator model with the allee effect," Ecological Complexity, vol. 11, pp. 12-27, 2012.

[22] R. López-Ruiz and D. Fournier-Prunaret, "Indirect Allee effect, bistability and chaotic oscillations in a predator-prey discrete model of logistic type," Chaos, Solitons \& Fractals, vol. 24, no. 1, pp. 85-101, 2005.

[23] G. Wang, X.-G. Liang, and F.-Z. Wang, "The competitive dynamics of populations subject to an Allee effect," Ecological Modelling, vol. 124, no. 2-3, pp. 183-192, 1999.

[24] P. A. Stephens and W. J. Sutherland, "Consequences of the Allee effect for behaviour, ecology and conservation," Trends in Ecology and Evolution, vol. 14, no. 10, pp. 401-405, 1999.

[25] M. S. Fowler and G. D. Ruxton, "Population dynamic consequences of Allee effects," Journal of Theoretical Biology, vol. 215, no. 1, pp. 39-46, 2002.

[26] P. Jyoti Pal and P. K. Mandal, "Bifurcation analysis of a modified Leslie-Gower predator-prey model with Beddington-DeAngelis functional response and strong Allee effect," Mathematics and Computers in Simulation, vol. 97, pp. 123-146, 2014.

[27] A. Korobeinikov, "A Lyapunov function for Leslie-Gower predator-prey models," Applied Mathematics Letters, vol. 14, no. 6, pp. 697-699, 2001.

[28] Y. Song, S. Yuan, and J. Zhang, "Bifurcation analysis in the delayed Leslie-Gower predator-prey system," Applied Mathematical Modelling, vol. 33, no. 11, pp. 4049-4061, 2009.

[29] X. Liu and J. Wang, "Bogdanov-Takens and triple zero bifurcations of a delayed modified Leslie-Gower predator prey system," Abstract and Applied Analysis, vol. 2013, Article ID 605471, 8 pages, 2013.
[30] Z.-G. Song, B. Zhen, and J. Xu, "Species coexistence and chaotic behavior induced by multiple delays in a food chain system," Ecological Complexity, vol. 19, no. 2, pp. 9-17, 2014.

[31] S. Chen, J. Shi, and J. Wei, "Global stability and Hopf bifurcation in a delayed diffusive lesliegower predator-prey system," International Journal of Bifurcation and Chaos, vol. 22, no. 3, Article ID 1250061, 2012.

[32] Z. Lu and X. Liu, "Analysis of a predator-prey model with modified Holling-Tanner functional response and time delay," Nonlinear Analysis: Real World Applications, vol. 9, no. 2, pp. 641-650, 2008.

[33] M. Kuussaari, I. Saccheri, M. Camara, and I. Hanski, "Allee effect and population dynamics in the Glanville fritillary butterfly," Oikos, vol. 82, no. 2, pp. 384-392, 1998.

[34] F. Wang and G. Pang, "Chaos and Hopf bifurcation of a hybrid ratio-dependent three species food chain," Chaos, Solitons and Fractals, vol. 36, no. 5, pp. 1366-1376, 2008.

[35] W. M. Liu, "Criterion of Hopf bifurcations without using eigenvalues," Journal of Mathematical Analysis and Applications, vol. 182, no. 1, pp. 250-256, 1994.

[36] A. Erbach, F. Lutscher, and G. Seo, "Bistability and limit cycles in generalist predator-prey dynamics," Ecological Complexity, vol. 14, pp. 48-55, 2013. 


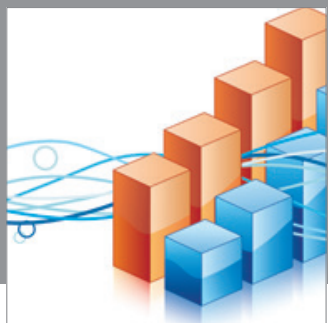

Advances in

Operations Research

mansans

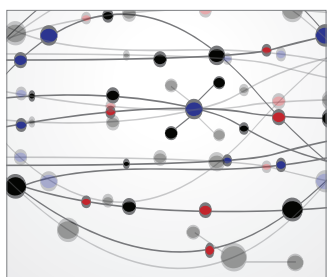

The Scientific World Journal
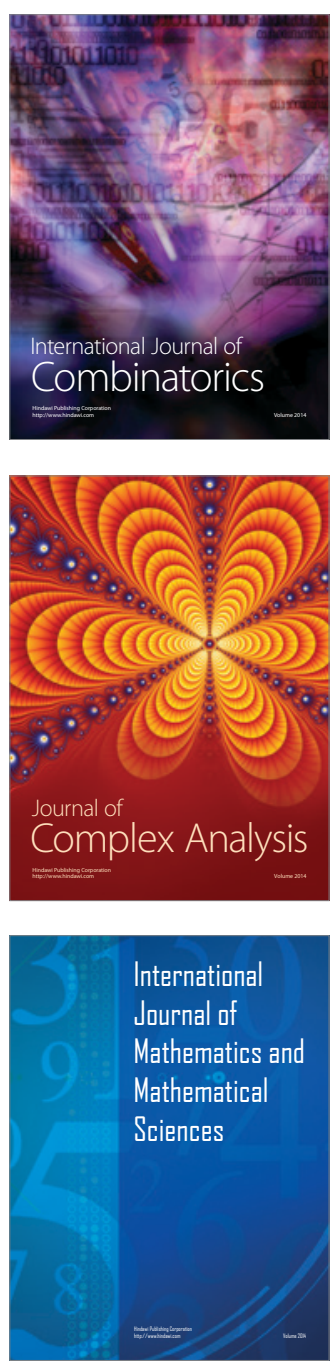
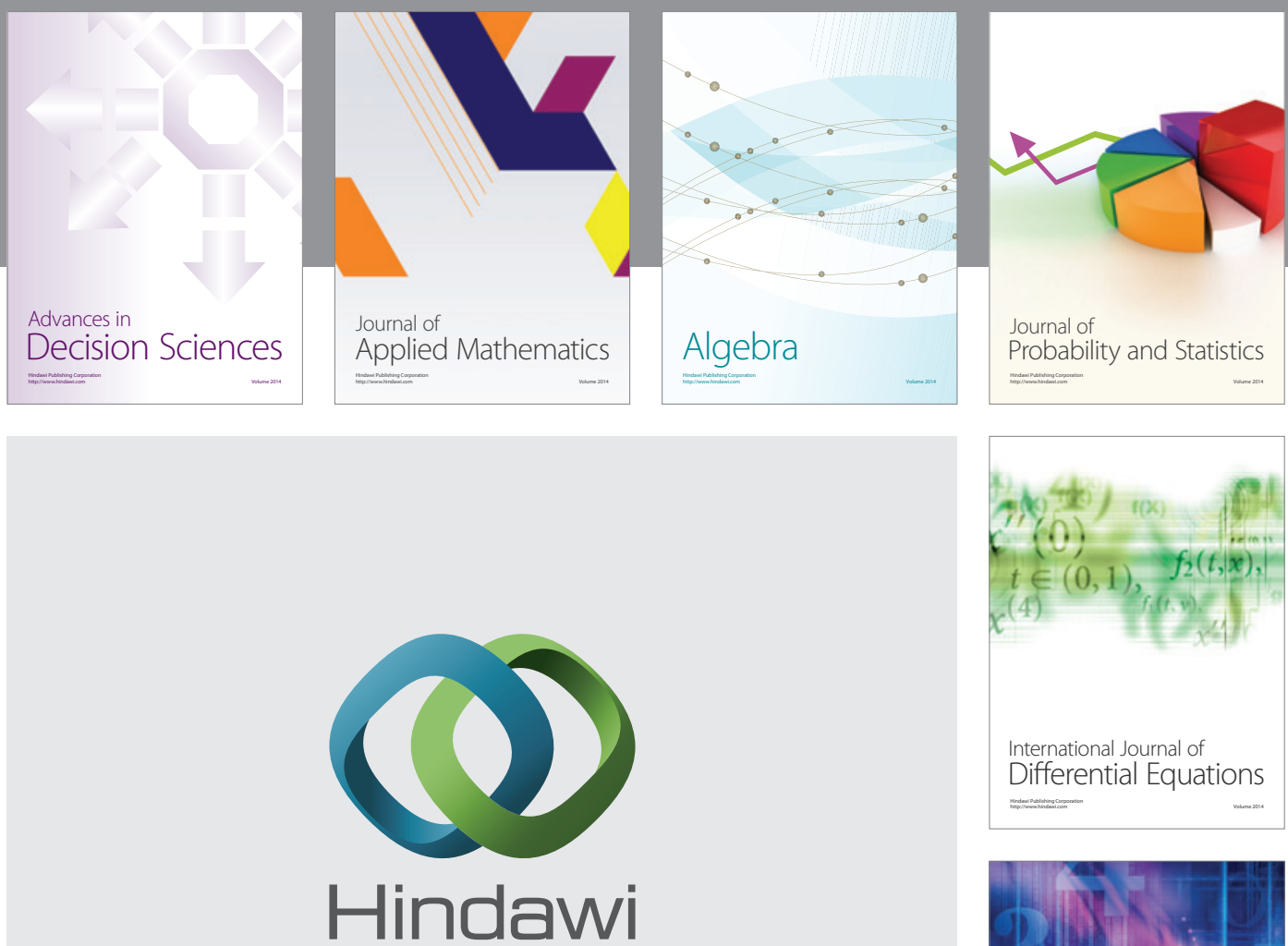

Submit your manuscripts at http://www.hindawi.com
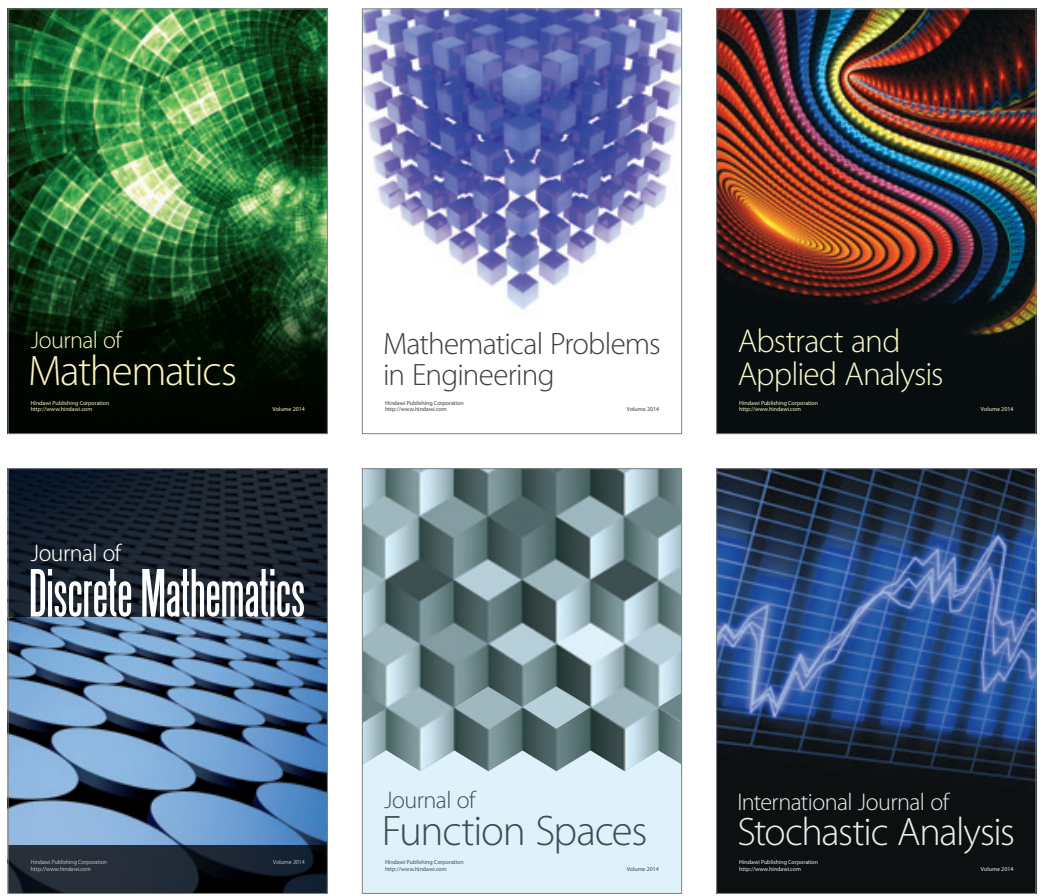

Journal of

Function Spaces

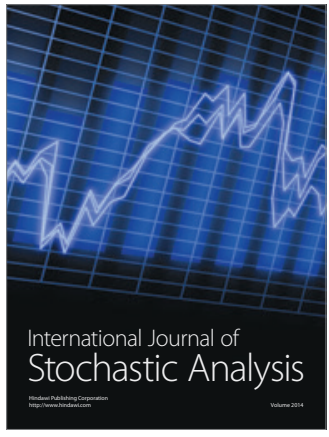

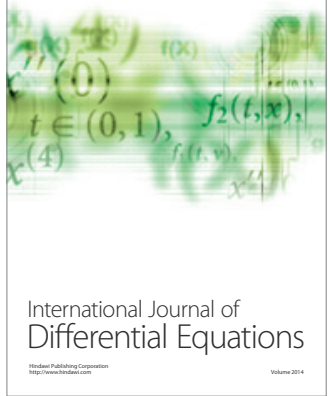
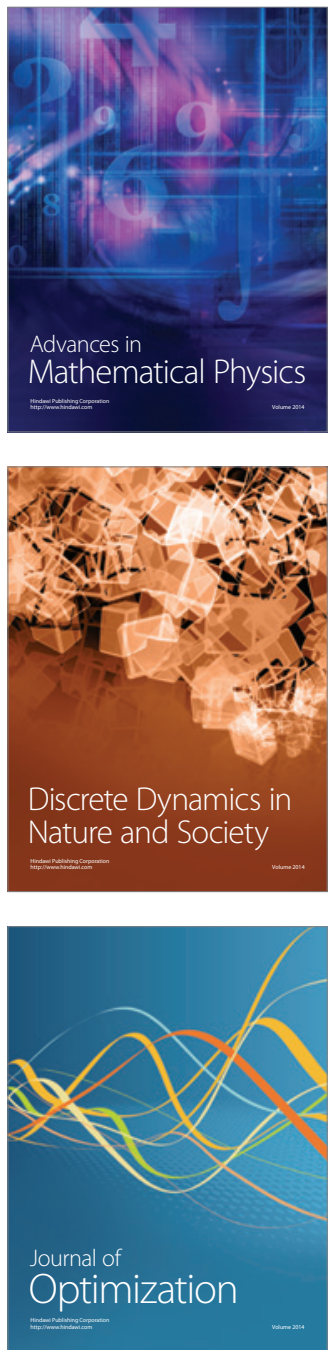